\title{
BMJ Open Association between cardiometabolic risk factors and body mass index, waist circumferences and body fat in a Zanzibari cross-sectional study
}

\author{
Maria Adam Nyangasa, ${ }^{1}$ Christoph Buck, ${ }^{1}$ Soerge Kelm, ${ }^{2}$ Mohammed Ali Sheikh, ${ }^{3}$
} Kim Laura Brackmann, ${ }^{1}$ Antje Hebestreit ${ }^{1}$

To cite: Nyangasa MA, Buck C, Kelm S, et al. Association between cardiometabolic risk factors and body mass index, waist circumferences and body fat in a Zanzibari crosssectional study. BMJ Open 2019;9:e025397. doi:10.1136/ bmjopen-2018-025397

\section{- Prepublication history for} this paper is available online. To view these files, please visit the journal online (http://dx.doi. org/10.1136/bmjopen-2018025397).

Received 13 July 2018 Revised 16 April 2019 Accepted 17 May 2019

Check for updates

(C) Author(s) (or their employer(s)) 2019. Re-use permitted under CC BY-NC. No commercial re-use. See rights and permissions. Published by BMJ.

${ }^{1}$ Leibniz Institute for Prevention Research and EpidemiologyBIPS, Bremen, Germany

${ }^{2}$ Centre for Biomolecular Interactions Bremen, Faculty for Biology and Chemistry, Bremen University, Bremen, Germany ${ }^{3}$ Environmental Analytical Chemistry and Eco-toxicology Lab, State University of Zanzibar, Zanzibar, United Republic of Tanzania

Correspondence to Dr. Antje Hebestreit; hebestr@leibniz-bips.de

\section{ABSTRACT}

Objectives To determine the prevalence of obesity indices (body mass index (BMI), waist circumference (WC), body fat per cent (BF\%)) and cardiometabolic risk factors. To investigate the association between obesity indices and cardiometabolic risk factors in a Zanzibari population.

Designs Cross-sectional study.

Settings Participants randomly selected from 80 Shehias (wards) in Unguja, Zanzibar in 2013.

Participants A total of 470 participants between 5 and 95 years were examined. Data on socioeconomic status, area of residence, anthropometry and venous blood were collected. Associations between obesity indices and cardiometabolic risk factors were investigated using multilevel logistic regression analyses in two steps: first, each obesity indicator was tested independently; second, all indicators combined in one model were tested for their association with cardiometabolic risk factors.

Results The proportion of overweight/obese individuals was $26.4 \%$, high WC (24.9\%) and high BF\% (31.1\%). Cardiometabolic risk factors with highest prevalence of abnormal values included hypertension (24.5\%), low high-density lipoprotein cholesterol (HDL-C) (29.4\%), high low-density lipoprotein cholesterol (LDL-C) (21.3\%) and high glycated haemoglobin (HbA1c) (19.1\%). Obesity and hypertension increased with age, and were most prevalent in participants aged 45 years and above. Low HDL-C was most prevalent among participants aged $\geq 18$ to $<45$ years, while high LDL-C was more prevalent in those above 45 years. High WC and high BF\% were associated with high levels of LDL-C (OR=2.52 (95\% Cl 1.24 to 5.13), $\mathrm{OR}=1.91$ (95\% Cl 1.02 to 3.58), respectively). Additionally, $\mathrm{BMI}$ and WC were associated with high levels of $\mathrm{HbA} 1 \mathrm{C}$ $(\mathrm{OR}=2.08(95 \% \mathrm{Cl} 1.15$ to 3.79$), \mathrm{R}=3.01(95 \% \mathrm{Cl} 1.51$ to 6.03 ), respectively). In the combined regression model, WC was associated with higher chances for hypertension $(\mathrm{OR}=2.62(95 \% \mathrm{Cl} 1.14$ to 6.06$))$ and for high levels of $\mathrm{HbA1c}(\mathrm{OR}=2.62(95 \% \mathrm{Cl} 1.12$ to 6.15$))$.

Conclusion High BMI, WC and $\mathrm{BF} \%$ were strongly associated with hypertension, with individuals with high WC being twice more likely to have hypertension; this calls for early and effective screening strategies for this study population.
Strengths and limitations of this study

- This is the first study to report the associations between obesity indices and cardiometabolic risk factors in Zanzibar.

- The household-based approach, which involved visiting the families in the home setting, resulted in a high individual response rate, thus minimising risk of selection bias.

- The cross-sectional design prevents us from drawing conclusions regarding the impact of changes in obesity indices on risk factors.

- Bioelectrical impedance analysis was used to estimate body fat percentage, which might have underestimated adiposity in children.

\section{INTRODUCTION}

Worldwide, cardiovascular diseases (CVDs) are the leading cause of death, ${ }^{1}$ and are emerging as a notable public health problem in sub-Saharan African countries. ${ }^{2}$ These countries are undergoing epidemiological transitions from communicable to non-communicable diseases (NCDs) that have been closely linked to increased urbanisation and rural-urban migration, which has led to unhealthy behaviours, including poor dietary habits and sedentary lifestyles. ${ }^{2}{ }^{3}$ According to the International Diabetes Federation (IDF), about 12 million people in Africa are estimated to have type 2 diabetes mellitus, ${ }^{4}$ with the prevalence ranging from $1 \%$ in rural Uganda to $12 \%$ in urban Kenya. ${ }^{5} 6$ Overweight and obesity have been found to be modifiable risk factors for cardiometabolic and other chronic diseases ${ }^{7}$ including hypertension, ${ }^{8}$ diabetes ${ }^{9}$ and dyslipidemia. ${ }^{10}$ The third report of the National Cholesterol Education Programme-Adult treatment Panel (NCEP-ATP III) also identified central obesity, dyslipidemia (hypertriglyceridemia and low levels of high-density lipoprotein 
cholesterol (HDL-C)), impaired glucose tolerance and elevated blood pressure as cardiometabolic risk factors. ${ }^{11}$

Multiple obesity indices such as body mass index (BMI), waist circumference (WC), body fat per cent $(\mathrm{BF} \%)$ and waist-to-hip ratio have been widely used to screen individuals for cardiometabolic risk in clinical and research settings ${ }^{12-14}$ due to their low cost and ease of administration. The performance of anthropometric indices may however vary according to different factors, including ethnicity, age, geographical area and population. ${ }^{13} 15$ BMI, which is based on weight and height, is the most widely used marker to assess body mass. In children and adolescents, the z-scores are used to classify obesity status, ${ }^{16}$ which is linked to metabolic risk, for example, in South African youth. ${ }^{17}$ However, BMI does not distinguish well between lean mass and fat mass. ${ }^{18}$ In contrast, WC is a measure of total body and abdominal fat accumulation and is better correlated with visceral adipose tissues than BMI. The correlation on the other hand varies significantly across ethnicities. ${ }^{19}{ }^{20}$ Another approach for measuring body fat is through bioelectrical impedance analysis (BIA), which has also been done in several epidemiological studies. ${ }^{21}$ The use of different anthropometric measurements might also provide complementary information which can be used to aid screening for cardiometabolic risk in different population settings. ${ }^{22} 23$

Few studies have investigated the performance of different obesity indices in association with cardiometabolic risk factors in sub-Saharan African populations. ${ }^{2718}$ Data from mainland Tanzania have shown an increasing prevalence of overweight and obesity in urban, periurban and rural areas. ${ }^{24}$ However, there is still a dearth of population-based studies investigating the associations of cardiometabolic risk factors with obesity indices in Tanzania mainland and Zanzibar. To help fill this gap, this study uses cross-sectional data of 470 individuals between 5 and 95 years who were examined in 2013 in Unguja Island, Zanzibar, to describe the prevalence of overweight/obesity and cardiometabolic risk factors in three age groups ( $\geq 5$ to $<18$ years, $\geq 18$ to $<45$ years and 45 years and above). The aim of the study was to identify vulnerable groups in the Zanzibari population with respect to cardiometabolic risk. Consequently, we investigated the association of $\mathrm{BMI}, \mathrm{WC}$ and $\mathrm{BF} \%$ with cardiometabolic risk factors (hypertension, total cholesterol, triglycerides, HDL, low-density lipoprotein (LDL), glycated haemoglobin (HbA1c), fasting plasma glucose and homeostasis model assessment of insulin resistance (HOMA-IR)). We considered the three obesity indices independently as well as combined, thereby reflecting different aspects of body composition.

\section{SUBJECTS AND METHODS}

\section{Study population and design}

We conducted a cross-sectional survey from September to December 2013 in a representative population sample in Unguja Island, Zanzibar. A total of 239 households were randomly selected and all household members were invited for the examination. As we also aimed to identify vulnerable groups within the families, we included young children and the elderly, who both normally depend on the family food environment. A two-stage sampling approach was used: (1) from a list of all 213 Shehias (wards), 80 Shehias were randomly selected; (2) households were randomly selected based on the Shehias' registration records. Participation agreement was requested from all members of a household. A total of 1443 family members agreed to participate and completed anthropometric and blood pressure measurements, as well as interviewer-administered questionnaires. Venous blood was also collected. The subgroup examinations are described in detail below. The complete description of the study design and methods has been described in detail elsewhere. ${ }^{25}$ The study was performed according to the Helsinki Declaration and the study protocol was evaluated and approved by the Ethics Committees of the University of Bremen and of the Zanzibar Ministry of Health and the Zanzibar Medical Research and Ethics Committee. All participants gave written informed consent and parents/guardians consented on behalf of their children in writing.

\section{Patient and public involvement}

During the development of the survey tools, measurements and the study protocol, a meeting was held with the local partners, government officials and researchers in Zanzibar to discuss the needs and gaps of the nutrition and health survey planned in Zanzibar. The documents and instruments were then modified according to the needs of the Zanzibari population as recommended in the meeting.

A year after the survey, preliminary results on the major health outcomes and related risk factors were presented and discussed during 2 days feedback workshop with the administrative leaders (e.g., Shehas, district commissioners), stakeholders (from health services, government officials, food safety) and our local partners in Zanzibar (academics and research). Each Sheha was handed a poster of the preliminary results, which was then displayed at their local offices for all Shehia members to see. District commissioners received a summary report on all Shehias of their districts. The preliminary results were further publicised on TV and print media. The same group of workshop participants was invited to a further workshop in 2018, whose aim was to identify target populations and channels for future nutrition education to address the aetiology and prevention of NCDs in the Zanzibari population, taking into consideration the survey results presented also in this study.

This observational epidemiological study examined participants in their home environment and did not enrol clinical patients.

\section{Questionnaires and anthropometric measurements}

Questionnaires were developed in English, translated into Swahili and then back translated to control for 
translation errors. Trained field staff collected the survey information. Parents reported their age and sex, as well as that of their children. Age was grouped into three categories $\geq 5$ to $<18$ years, $\geq 18$ to $<45$ years and 45 years and above. In addition, parental highest educational level according to the International Standard Classification of Education (ISCED) ${ }^{26}$ was used as a proxy indicator for socioeconomic status of the family. Education was categorised into low education (no education and primary school) and high education (secondary school and above). To determine participants' area of residence, information on region, district and Shehia (the smallest administrative unit in Zanzibar) was recorded and two categories for area of residence were developed (urban and rural). Utilisation of medication was also documented in the questionnaire. Regarding medication for obesity-related conditions, participants reported use of hypertension medication but not of diabetes or dyslipoproteinemia medication. Hence, the variable was later categorised as 'hypertension medication' and 'other medication' (eg, antimalaria therapy or antipyretic products). To ensure a high quality of data collection, this study used proven examination methods and laboratory standards. ${ }^{27} 28$ All anthropometric measurements and physical examinations were adopted from the Identification and prevention of dietary- and lifestyle induced health effects in Children and infants (IDEFICS) study and conducted following standardised procedures. ${ }^{29}{ }^{30}$ Measurement of body weight was carried out to the nearest $0.1 \mathrm{~kg}$ and $\mathrm{BF} \%$ was determined using the BIA method using an electronic scale (TANITA BC-420 SMA, Germany). Height was measured using a SECA 213 stadiometer, UK, and WC was measured midway between the lowest rib and the iliac crest, using an inelastic measuring tape (SECA 201). For all measurements, participants wore light clothing. The measures were recorded to the nearest $0.1 \mathrm{~cm}$. The complete description of the anthropometric measurements of the study is described elsewhere. ${ }^{25}$

For children and adolescents, BMI was calculated as $\mathrm{kg} / \mathrm{m}^{2}$ and then transformed to age-specific and sex-specific z-score and percentiles. Thereafter, categories for overweight (BMI between $>75$ th and $<95$ th percentile) and obesity (BMI $>95$ th percentile) were built according to the WHO centile curves. ${ }^{16}{ }^{31}$ For adults, overweight/ obesity was defined as BMI $\geq 25 \mathrm{~kg} / \mathrm{m}^{2}$ as recommended by WHO. ${ }^{32}$ For statistical analysis, the BMI categories were merged into two: (1) underweight/normal weight $(\leq 75$ th percentile for children and adolescents and $<25 \mathrm{~kg} / \mathrm{m}^{2}$ for adults) and (2) overweight/obesity ( $>75$ th percentile and $\geq 25 \mathrm{~kg} / \mathrm{m}^{2}$ ). Regarding WC, high abdominal obesity was defined as $\mathrm{WC} \geq 90$ th percentile for children below 10 years ${ }^{33}$; WC $\geq 90$ th percentile for adolescents aged 10 to $<16$ years and $\mathrm{WC}>94 \mathrm{~cm}$ for men and $>80 \mathrm{~cm}$ for women for participants 16 years and older, as recommended by the IDF. ${ }^{34}$ As recommended by McCarthy $e t$ $a l,{ }^{35}$ for boys and girls below 18 years, high BF\% was set at $\geq 85$ th percentile. For adults above 18 years, high BF\% was defined as $\geq 20 \%$ for men and $\geq 32 \%$ for women. ${ }^{36}$ The cut-offs and references are listed in table 1.

\section{Cardiometabolic risk factors}

All blood samples were drawn after overnight fasting and were collected from all eligible participants over 5 years of age by venepuncture.$^{37}$ To reduce pain, children below 10 years of age were given a local anaesthetic plaster before blood drawing, which motivated the children to participate. Before blood drawing, the procedure was once again explained to all participants in easy language and they were informed that they still could refuse to participate. For children weighing $10 \mathrm{~kg}$, the blood collection was restricted to $1 \%$, corresponding to approximately $8 \mathrm{~mL}$. For healthy, non-pregnant adults weighing at least $50 \mathrm{~kg}$, a maximum of $20.5 \mathrm{~mL}$ venous blood was drawn. Collection, processing and storage of blood samples are described elsewhere. ${ }^{25}$

Metabolic parameters were categorised for investigating the prevalence of cardiometabolic disorders in the study population. Due to the wide range of age groups in this study population, different cardiometabolic risk definitions and cut-offs were used (table 1). Cardiometabolic risk for children between 5 and 10 years was defined according to age-specific and sex-specific cut-offs. The parameters, including hypertension (systolic blood pressure and diastolic blood pressure), blood lipids (high total cholesterol (TC), high triglycerides (TG), LDL cholesterol (LDL-C) and HDL-C), blood glucose/insulin (HOMA-IR and elevated fasting plasma glucose (FPG), were defined according to the IDEFICS study. ${ }^{38-40}$ HbAlc was defined according to Rödöö $e t a l^{41}$ for children under 17 years. For children and adolescents between 10 and 16 years, hypertension was defined according to age-specific and sex-specific cut-offs as recommended ${ }^{42}$; for adolescents and adults >16years hypertension was defined as recommended. ${ }^{43}$ Blood lipids (TC and LDL-C) were defined according to the NCEP ${ }^{11}$ and TG, HDL-C and FPG according to IDF. ${ }^{34}$ HbAlc for participants $>17$ years was defined according to Stern $e t a t^{44}$ and insulin resistance was estimated as HOMA-IR according to the reference value of HOMA-IR as recommended by Shashaj $e t a l^{45}$ In the present study, the 75 th percentile cut-off was used for children and adolescents from 10 to 17 years. For participants $>17$ years, HOMA-IR was defined according to von Eyben et al. ${ }^{46}$ HOMA-IR was calculated from glucose $(\mathrm{mmol} / \mathrm{L}))$ and insulin $(\mu \mathrm{U} / \mathrm{mL})$ concentrations using the formula: HOMA-IR $=$ (fasting insulin $\times$ fasting glucose/22.5). ${ }^{47}$

\section{Inclusion criteria for study sample}

Of 1443 individuals who participated in this study, 1314 fulfilled the inclusion criteria (age, sex, weight, height) for the overall study analysis. Of the 1314 participants, 1234 provided complete $\mathrm{WC}$ and $\mathrm{BF} \%$ measurements. Among these, 557 provided complete blood samples for the cardiometabolic risk analysis and only 505 were on fasting status. To reduce bias while estimating mean and 
Table 1 Cardiometabolic risk definitions and references

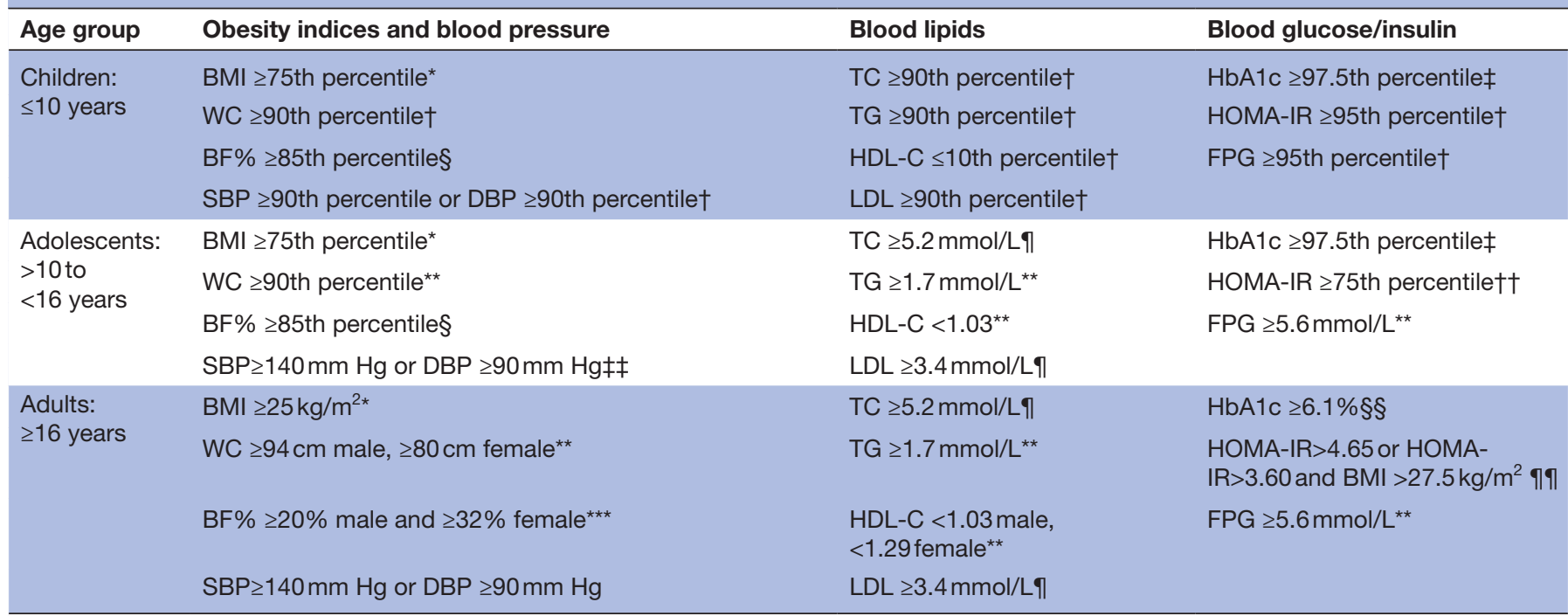

*WHO.

†IDEFICS study.

†Rödöö et al. ${ }^{41}$

§McCarthy et al. ${ }^{35}$

ףNational Cholesterol Education Programme.

${ }^{* *}$ International Diabetes Federation.

††Shashaj et $\mathrm{a}{ }^{45}$ for children and adolescents under 17 years.

$\ddagger \ddagger$ National Institute of Health third and seventh report, respectively.

$\S \S S t e r n$ et $a l^{44}$ for adults above 17 years.

ๆीvon Eyben et al. ${ }^{46}$

${ }^{\star * \star}$ Gallagher et al. ${ }^{36}$

BF\%, body fat per cent; BMI, body mass index; DBP, diastolic blood pressure; FPG, fasting plasma glucose; HbA1c, glycated haemoglobin;

HDL-C, high-density lipoprotein cholesterol; HOMA-IR, homeostasis model assessment of insulin resistance; LDL-C, low-density

lipoprotein cholesterol; SBP, systolic blood pressure; TC, total cholesterol; TG, triglyceride; WC, waist circumference.

$\mathrm{SD}$ in the regression analysis, we excluded the top $1 \%$ of individuals with extremely high values for cardiometabolic risk and obesity indices, leaving us with a complete sample of 470 participants for the analysis.

\section{Statistical analysis}

Descriptive analysis was conducted to calculate the mean $\mathrm{SD}$ and range (minimum, maximum) for continuous variables, as well as the distribution of the categorical data in $\mathrm{N}$ and percentages (\%). As part of the regression analysis, we tested the necessary assumptions in terms of symmetry and normality using residual plots and $Q-Q$ plots. Mixed logistic regression models were used to analyse the association between obesity indices and cardiometabolic risk factors. In addition, potential clustering within households was considered in terms of a random intercept. Following the hierarchy of the municipal structure in Zanzibar, we conducted sensitivity analysis modelling either Shehias or households within Shehias as a random intercept in the models. Since the results of the models only showed marginal differences, we only considered the household as a random intercept in our analyses. First, mixed logistic regression models were conducted to estimate the association between each of the three obesity indicators (BMI, $\mathrm{WC}$ and $\mathrm{BF} \%$ ) as exposure variables and each of the eight risk factors (hypertension, TC, TG, HDL-C, LDL-C,
HbA1c, FPG and HOMA-IR) as dependent variables, in terms of ORs and $95 \%$ CIs. Since BMI, WC and $\mathrm{BF} \%$ are inter-related, the strongest relationship with cardiometabolic risk factors was investigated by conducting mixed logistic regression models. This was done by estimating the association (ORs and 95\% CIs) between all three obesity indices as dependent variables in one model and each of the eight risk factors as outcome variables. All models were adjusted for potential confounders and covariates such as gender, age, education level (ISCED), area of residence and utilisation of hypertension medication. Statistical analysis was performed using SAS V.9.3 (SAS Institute, Cary, North Carolina, USA); mixed logistic regression models were conducted based on the GLIMMIX procedure; statistical significance was set at $\alpha=0.05$.

\section{RESULTS}

\section{Distribution of obesity and cardiometabolic risk and} characteristics of the study population by age groups $(n=470)$ The mean age was $29( \pm 18)$ years, with the highest proportion being in the age group ( 218 to $<45$ years) (table 2). The overall mean values for BMI, WC and $\mathrm{BF} \%$ were as follows: BMI $22 \mathrm{~kg} / \mathrm{m}^{2}( \pm 5.2)$, WC $75 \mathrm{~cm}( \pm 16)$ 
Table 2 Distribution of obesity and cardiometabolic risk in the study population $(n=470)$ by age group (means and SD)

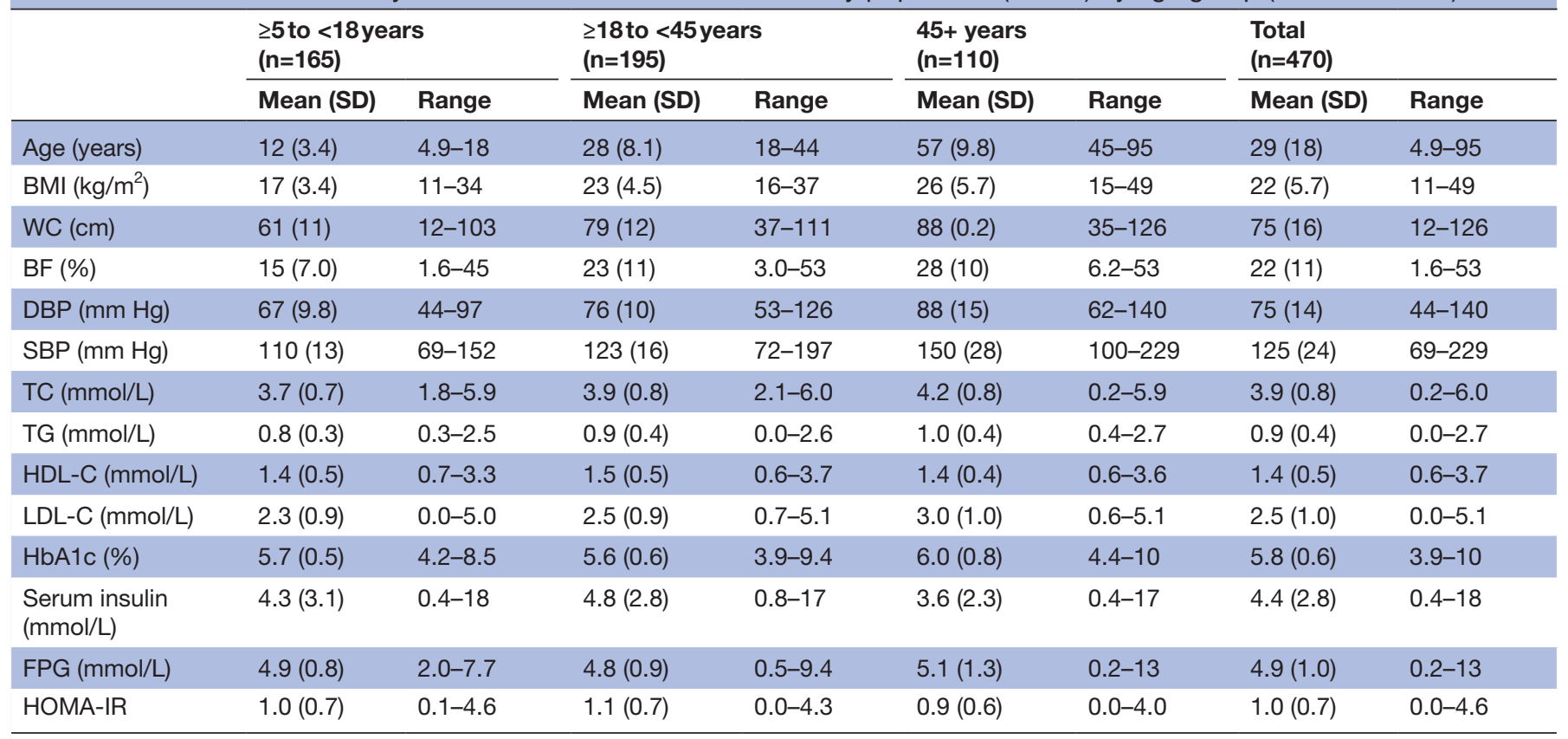

BF\%, body fat per cent; BMI, body mass index; DBP, diastolic blood pressure; FPG, fasting plasma glucose; HbA1c, g lycated haemoglobin; HDL, high-density lipoprotein; HOMA-IR, homeostasis model assessment of insulin resistance; LDL, low-density lipoprotein; SBP, systolic blood pressure; TC, total cholesterol; TG, triglyceride; WC, waist circumference.

and BF\% 22\% $( \pm 11)$. The mean BMI of $26 \mathrm{~kg} / \mathrm{m}^{2}( \pm 5.7)$ for participants $>45$ years was slightly higher than normal, indicating overweight. Mean diastolic blood pressure was in the normal range for all the age groups, but a higher mean value of systolic blood pressure, $150 \mathrm{~mm} \mathrm{Hg}$ $( \pm 280)$ was observed among participants $>45$ years. The mean values of most of the variables showed an increase with age group, except for HDL-C and diabetes markers (HbAlc, serum insulin, plasma glucose and HOMA-IR), which showed no specific trend.

Of the 470 participants, more than half were women: $52.6 \%$ ( $\mathrm{n}=247), 51.9 \%$ (244) had higher education level and $73.4 \%$ (345) resided in urban area. Regarding education level, the majority of those with higher education level were aged $\geq 18$ to $<45$ years $(150 / 244=61 \%)$ (table 3$)$.

The overall proportion of overweight/obesity with regard to $\mathrm{BMI}$, $\mathrm{WC}$ and $\mathrm{BF} \%$ was $26.4 \%, 24.9 \%$ and $31.1 \%$, respectively, and increased with age (table 2). The highest proportion was observed among participants $>45$ years. We observed different trends in the prevalence of metabolic parameters and hypertension across age groups. The prevalence of hypertension, high TC, LDL-C and HbA1c increased with age, while that for TGs and HOMA-IR decreased with age. The most prevalent factors were reduced HDL-C (29.4\%), hypertension $(24.5 \%)$ as well as raised LDL-C (21.3\%) and HbAlc levels (19.1\%). Although hypertension was more prevalent among participants $>45$ years, only about $9.4 \%$ (10) of the participants in this age group were on hypertension medication. Furthermore, high LDL-C and HbA1c were more prevalent among participants $>45$ years, and low HDL-C was most prevalent among those aged $\geq 18$ to $<45$ years.

\section{Association between obesity indices and cardiometabolic risk} factors

Obesity indices (BMI, WC and $\mathrm{BF} \%$ ) were observed to be associated with one or more risk factors. Participants with high BMI (OR=2.41 (95\% CI 1.33 to 4.47$)$ ), high WC $(\mathrm{OR}=3.68(95 \%$ CI 1.81 to 7.52$))$ or high $\mathrm{BF} \%$ (OR=2.51 (95\% CI 1.40 to 4.51$)$ ) were more likely to be hypertensive (table 4). Having high WC (OR=2.52 (95\% CI 1.24 to 5.13)) or high $\mathrm{BF} \%(\mathrm{OR}=1.91$ (95\% CI 1.02 to 3.58$)$ ) was associated with higher chances of having high LDL-C. Furthermore, BMI (OR=2.08 (95\% CI 1.15 to 3.79)) and WC $(\mathrm{OR}=3.01$ (95\% CI 1.51 to 6.03)) were associated with HbA1c levels. We further observed increased OR for obesity indices with regard to high TC, high TG, low HDL-C, elevated glucose and HOMA-IR. As the proportion of individuals with high HOMA-IR was very small in our sample $(1.28 \% / \mathrm{n}=6)$, the results were not considered in the final regression analysis. Regarding goodness of fit of the models, values of the Akaike Information Criterion, which estimates the quality of each model relative to that of each of the other models, showed that models including WC as an obesity index tended to have a slightly stronger relationship compared with those including BMI and $\mathrm{BF} \%$.

Table 5 presents results of mixed logistic regression models including all three obesity indices to investigate the association with single cardiometabolic risk factors. Compared with the separate regression models, the ORs for most of the associations were attenuated. However, having high WC was again associated with a higher chance of having hypertension (OR=2.62 (95\% CI 1.14 to 6.06$))$ and having high HbA1c levels $(\mathrm{OR}=2.62(95 \%$ 
Table 3 Characteristics of the study population $(n=470)$ by age group $(n / \%)$

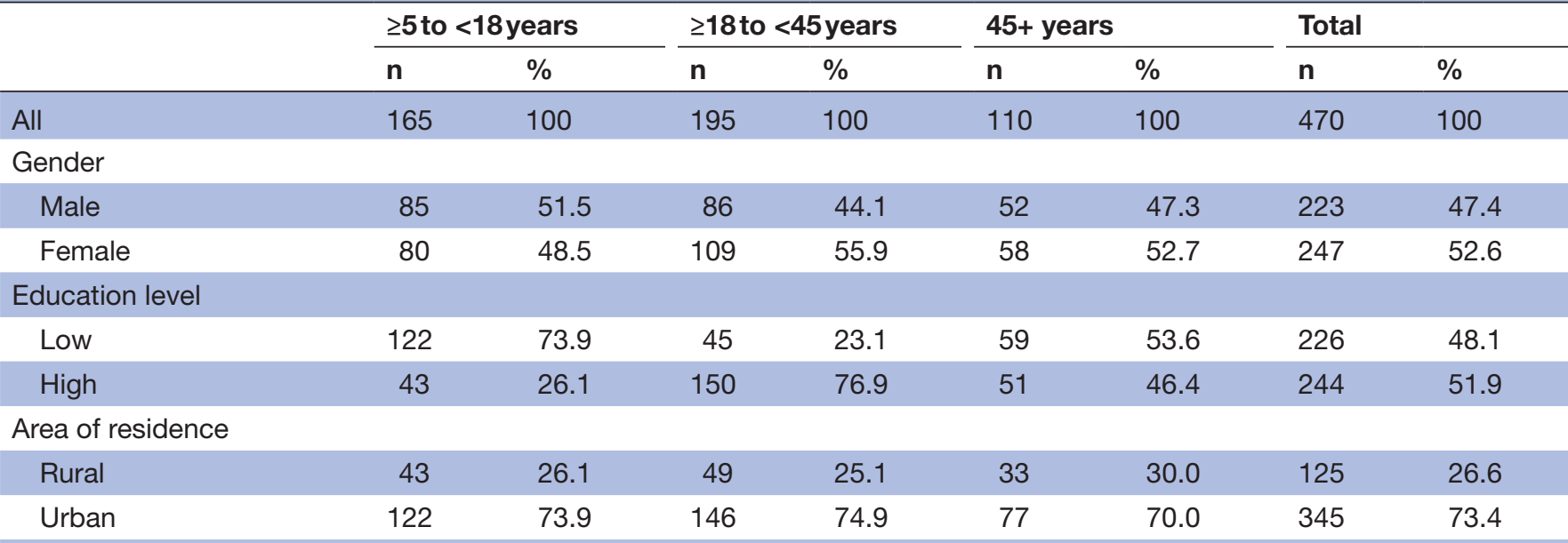

\section{Obesity indices}

BMI

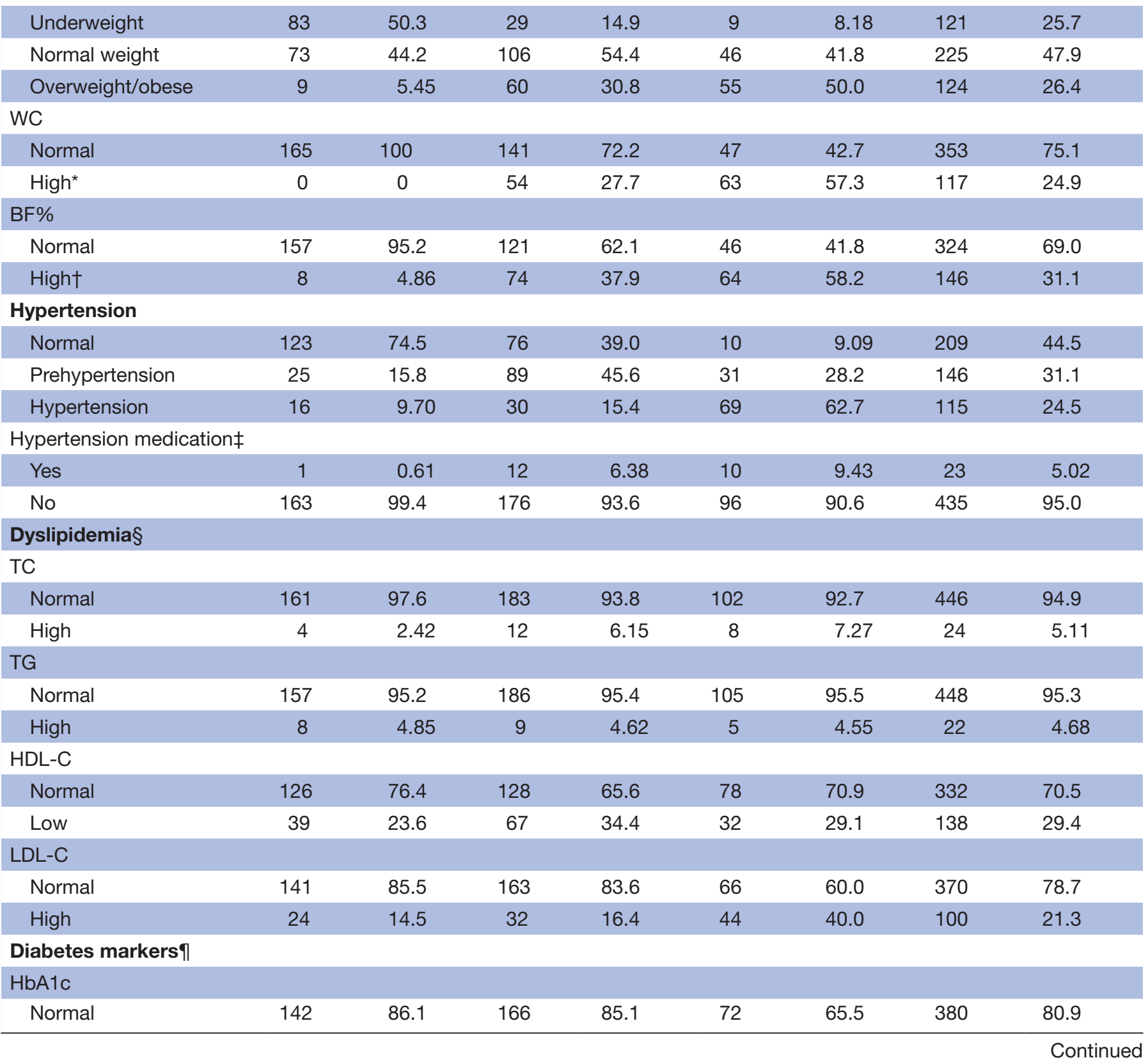


Table 3 Continued

\begin{tabular}{|c|c|c|c|c|c|c|c|c|}
\hline & \multicolumn{2}{|c|}{$\geq 5$ to $<18$ years } & \multicolumn{2}{|c|}{$\geq 18$ to $<45$ years } & \multicolumn{2}{|c|}{$45+$ years } & \multicolumn{2}{|c|}{ Total } \\
\hline & $\mathbf{n}$ & $\%$ & $n$ & $\%$ & n & $\%$ & $n$ & $\%$ \\
\hline High & 23 & 13.9 & 29 & 14.9 & 38 & 34.5 & 90 & 19.1 \\
\hline \multicolumn{9}{|c|}{ Plasma glucose } \\
\hline Normal & 136 & 82.4 & 167 & 85.6 & 88 & 80.0 & 391 & 83.2 \\
\hline High & 29 & 17.6 & 28 & 14.4 & 22 & 20.0 & 79 & 16.8 \\
\hline \multicolumn{9}{|l|}{ HOMA-IR } \\
\hline Normal & 162 & 98.2 & 193 & 99.0 & 109 & 99.1 & 464 & 98.7 \\
\hline High & 3 & 1.82 & 2 & 1.03 & 1 & 0.91 & 6 & 1.28 \\
\hline
\end{tabular}

${ }^{*}$ High WC was defined as WC $\geq 90$ th percentile for children $<10$ years. ${ }^{33}$ Adolescents between 10 and 16 years and adults $>16$ years WC $>94 \mathrm{~cm}$ for men and $>80 \mathrm{~cm}$ for women according to IDF cut-off. ${ }^{34}$

†High BF\% for adults (overweight/obese) $\geq 20$ for men and $\geq 32$ for women according to (NIH/WHO) BMI guidelines ${ }^{36}$ and $\geq 85$ th percentile for children. ${ }^{35}$

¥Missing information from 12 participants $(n=458)$.

$\S$ High dyslipidemia for adults; was defined as total serum cholesterol $(\geq 6.2 \mathrm{mmol} / \mathrm{L})$ and LDL-C $(\geq 3.4 \mathrm{mmol} / \mathrm{L})$, ${ }^{11} \mathrm{low} \mathrm{HDL}-\mathrm{C}<1.03 \mathrm{mmol} / \mathrm{L}$ in men or $<1.29 \mathrm{mmol} / \mathrm{L}$ in women with hypertriglyceridemia $(\geq 1.7 \mathrm{mmol} / \mathrm{L})^{34}$ and for children according to IDEFICS study. ${ }^{39}$

१High diabetes risk markers; high HbA1c $(>6.1 \%),{ }^{46}$ high FPG $(\geq 5.6 \mathrm{mmol} / \mathrm{L})^{34}$ and $\mathrm{HOMA}-\mathrm{IR}>4.65$ or $>3.60$ and $\mathrm{BMI}>27.5 \mathrm{~kg} / \mathrm{m}^{2} 43$ and for children with high $\mathrm{HbA} 1 \mathrm{c}(\geq 97.5$ th percentile), high $\mathrm{FPG} \geq 95$ th percentile and HOMA-IR $\geq 95$ th percentile.

$\mathrm{BF} \%$, body fat per cent; BMI, body mass index; FPG, fasting plasma glucose; HbA1c, glycated haemoglobin; HDL-C, high-density lipoprotein cholesterol; HOMA-IR, homeostasis model assessment of insulin resistance; LDL-C, low-density lipoprotein cholesterol; TC, total cholesterol; TG, triglyceride; WC, waist circumference.

CI 1.12 to 6.15$)$ ). Again, as the proportion of individuals with high HOMA-IR levels was very small in our sample $(1.28 \% / \mathrm{n}=6)$, HOMA-IR was not considered in the final regression analysis.

\section{DISCUSSION}

This study is the first population-based survey in Unguja Island that investigated the association between multiple obesity indices (BMI, WC and $\mathrm{BF} \%$ ) and multiple cardiometabolic risk factors in a randomly selected Zanzibari population, aged 5-95 years. This study population, as in many other low-income and middle-income countries (LMICs), is undergoing a coexistence of the double burden of underweight children and overweight/obese adults. Generally, about a quarter of the study population were overweight/obese, and obesity increased with age. This observation has also been reported in demographic health surveys from seven sub-Saharan African countries. ${ }^{48}$ In the adult population, the prevalence of overweight/obesity was lower than that in Ghana, ${ }^{7}$ but higher than in Nigeria ${ }^{2}$ and Benin. ${ }^{49}$ On the other hand, $>50 \%$ of the children in this study were underweight, a proportion higher than that in other sub-Saharan African countries (Kenya, Nigeria, South Africa, Equatorial Guinea and Cameroon).$^{50}$

Dyslipidemia is a risk factor for a variety of cardiovascular diseases and is becoming more prevalent in sub-Saharan Africa, particularly the form of low HDL-C. ${ }^{51} 52$ Despite the relatively normal levels of TC and TG, low HDL-C affected about $29 \%$ of the overall population,

Table 4 Associations between obesity indices (independent) and cardiometabolic risk factors (dependent), adjusted for gender, age, education level, area of residence and hypertension medication $(n=470)$

\begin{tabular}{|c|c|c|c|c|c|c|c|c|c|}
\hline \multirow{2}{*}{$\begin{array}{l}\text { Obesity indices } \\
\text { Risk factors }\end{array}$} & \multicolumn{3}{|c|}{ High BMI } & \multicolumn{3}{|c|}{ High WC } & \multicolumn{3}{|c|}{ High BF\% } \\
\hline & OR & $95 \% \mathrm{Cl}$ & AIC & OR & $95 \% \mathrm{Cl}$ & AIC & OR & $95 \% \mathrm{Cl}$ & AIC \\
\hline Hypertension & 2.41 & 1.33 to 4.47 & 504.86 & 3.68 & 1.81 to 7.52 & 499.79 & 2.51 & 1.40 to 4.51 & 503.46 \\
\hline High TC & 1.13 & 0.40 to 3.19 & 192.74 & 0.84 & 0.27 to 2.66 & 192.71 & 1.05 & 0.37 to 2.95 & 192.79 \\
\hline High TG & 1.79 & 0.55 to 5.77 & 189.88 & 2.23 & 0.58 to 8.66 & 189.38 & 1.64 & 0.52 to 5.14 & 190.11 \\
\hline Low HDL-C & 1.21 & 0.62 to 2.37 & 516.08 & 1.15 & 0.55 to 2.42 & 516.25 & 1.06 & 0.54 to 2.05 & 516.37 \\
\hline High LDL-C & 1.45 & 0.78 to 2.69 & 457.62 & 2.52 & 1.24 to 5.13 & 452.23 & 1.91 & 1.02 to 3.58 & 454.77 \\
\hline High HbA1c & 2.08 & 1.15 to 3.79 & 442.70 & 3.01 & 1.51 to 6.03 & 438.53 & 1.75 & 0.96 to 3.18 & 445.23 \\
\hline High glucose & 2.04 & 0.93 to 4.50 & 397.36 & 2.07 & 0.84 to 5.07 & 397.98 & 1.76 & 0.80 to 3.87 & 398.56 \\
\hline
\end{tabular}

AIC, Akaike Information Criterion; BF\%, body fat per cent; BMI, body mass index; HbA1c, g lycated haemoglobin; HDL-C, high-density lipoprotein cholesterol; LDL-C, low-density lipoprotein cholesterol; TC, total cholesterol; TG, triglyceride; WC, waist circumference. 
Table 5 Associations between obesity indices (independent) and cardiometabolic risk factors (outcome) adjusted by gender, age, education level and area of residence $(n=470)$

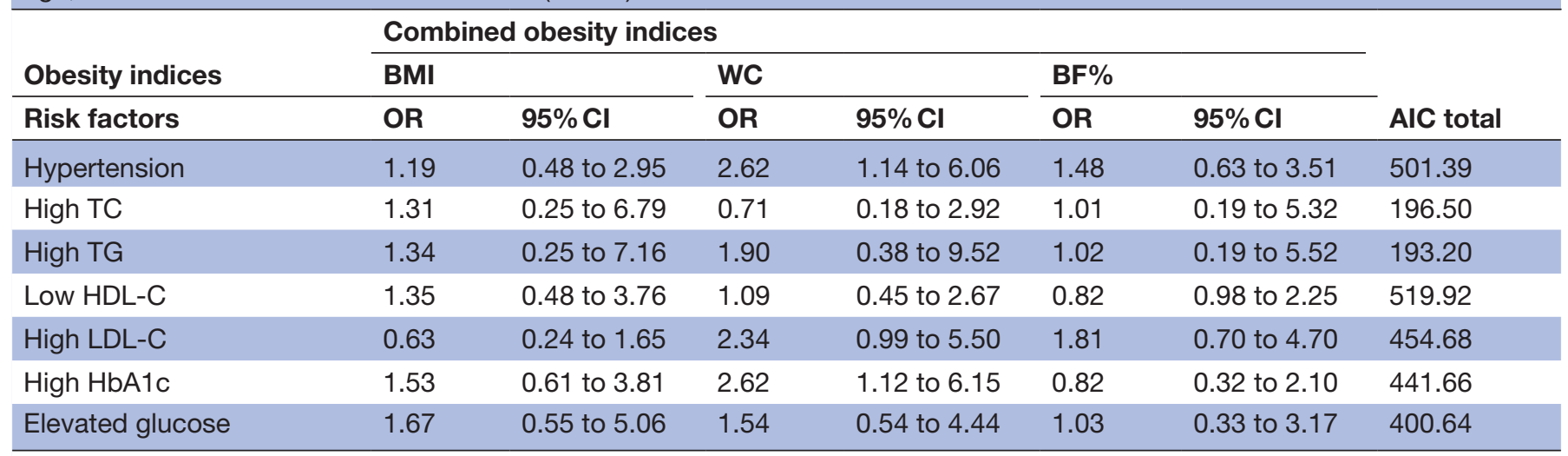

AIC, Akaike Information Criterion; BF\%, body fat per cent; BMI, body mass index; HbA1c, g lycated haemoglobin; HDL-C, high-density lipoprotein cholesterol; LDL-C, low-density lipoprotein cholesterol; TC, total cholesterol; TG, triglyceride; WC, waist circumference.

an indication that low HDL-C affects a large proportion of adults $>18$ years. The low HDL-C levels observed in our study population might therefore be indicative of a notable and evolving cardiovascular risk in the study region. Our results are in line with a recent study in sub-Saharan Africa and Middle East with $30 \%$ of the participants having low HDL-C. ${ }^{53}$ Other studies in sub-Saharan Africa reported even higher prevalence of low HDL-C, $43.1 \%$ in Nigeria ${ }^{2}$ and $80 \%$ in Botswana, ${ }^{54}$ mostly affecting individuals between 35 and 54 years.

In the present study, a high proportion of participants with high HbAlc (14\%) and elevated fasting glucose $(18 \%)$ are children below 18 years. Since diabetes in children in LMICs has not received much attention, it is likely that there is a high number of children with subclinical complications due to delayed or missed diagnosis as well as a lack of regular monitoring. The high proportions observed in this study are a possible indication that a large proportion of participants with diabetes are not aware of their status and are hence not monitored or treated. The fact that diabetes medication was not reported in this sample supports this assumption. However, when using WHO diabetes diagnostic criteria, ${ }^{55}$ that is, HbAlc cut-off $\geq 6.5 \%$ and $\mathrm{FPG} \geq 7.0 \mathrm{mmol} / \mathrm{L}$, the prevalence of diabetes in participants $>18$ years decreased to $8.14 \%$ and $3.05 \%$, respectively (data not shown). The most intriguing result however is the high proportion of children between 5 and $<18$ years being at high risk for diabetes with elevated FPG levels when using cut-off of $\geq 5.6 \mathrm{mmol} / \mathrm{L}$. Our results showed that the prevalence of FPG and HOMA-IR in children and adolescents $<18$ years was in general higher than that of adults $>18$ years, but less than that of adults $>45$ years. Results from previous cross-sectional studies have shown that physiological transient insulin resistance develops in children during puberty ${ }^{56}$ and decreases again by the end of puberty, regardless of obesity. The decrease in insulin sensitivity in the pubertal period is said to lead to an increase in glucose-stimulated insulin secretion. ${ }^{57}$ The high prevalence of FPG and HOMA-IR observed in children and adolescents in our study could hence be due to physiological changes in children and adolescents during prepubertal period and puberty. They could however also be due to misreporting (children did not report having eaten prior to the blood drawing), or to a true high risk within this age group. Considering this, we adjusted for age in the regression models in order to control for possible confounding effects of physiological changes through maturation and ageing. Interestingly, the prevalence of high FPG decreased from approximately $18 \%$ to $0.61 \%$ when we used the $\mathrm{WHO}^{55}$ diabetes diagnostic criteria (FPG $\geq 7 \mathrm{mmol} / \mathrm{L}$ ) for the same age group (data not shown). This, in our opinion, indicates that the majority of the children are at risk for diabetes, and that the cut-off for HbAlc $\geq 6.1 \%$ as well as elevated FPG $\geq 5.6 \mathrm{mmol} / \mathrm{L}$ seem to be better screening tools for identifying those at risk, earlier.

Our study showed a strong association between BMI, $\mathrm{WC}$ and $\mathrm{BF} \%$ and hypertension in the study population. These findings are in agreement with other studies that also reported an association between hypertension/ prehypertension, $\mathrm{BMI}$ and $\mathrm{WC}^{58}$ as well as $\mathrm{BF} \% .^{7}$ Moreover, the association between hypertension and high $\mathrm{WC}$ was twice as strong as that with high BMI and high $\mathrm{BF} \%$. This result suggests that central obesity may be a better indicator for the risk of hypertension and other cardiovascular diseases in our study population. Thus, optimal body weight control and reduced central obesity risk may have beneficial effects on hypertension control in this population. This study also observed a strong association between WC and LDL-C levels. Obirikorang et al also reported similar associations in a comparative cross-sectional study conducted in Ghana. ${ }^{7}$

In the separate models, strong associations were observed between BMI, WC and HbAlc levels, which can be explained by the inter-relation of the two indices, since abdominal fat accumulation increases in proportion to $\mathrm{BMI}^{59}$ and $\mathrm{BMI}$ is one of the main risk factors for diabetes and prediabetes. ${ }^{60}$ However, when all three obesity indices 
were combined, it is only the association between WC and HbA1c levels and hypertension that remained strong. Excessive visceral fat in abdominal obesity is the main source of free fatty acids and inflammatory cytokines, which, according to the literature, might lead to insulin resistance and type 2 diabetes mellitus. ${ }^{61}$ This probably explains why WC was strongly associated with diabetes and hypertension in our study population. Therefore, measuring WC using optimal WC cut-off values as was done in this study would be a feasible, less time consuming and cost-effective screening tool to identify at-risk individuals in the Zanzibari population.

This study has some limitations that should be considered. First, this study investigated the association between obesity indices and cardiometabolic risk factors using cross-sectional data; thus, we were not able to examine the impact of changes in obesity indices on risk factors. Second, as is done in many epidemiological studies and clinical trials, we used BIA to estimate $\mathrm{BF} \%$. However, compared with skinfold measurements, BIA measurements may underestimate adiposity in children. ${ }^{62}$ Third, even though we excluded participants who reported food or beverage intake prior to blood drawing during the data cleaning process, we cannot entirely rule out misreporting of the 'fasting status'. The overall aim of the study was to estimate the prevalence of malnutrition in the Zanzibari population including possible correlates. Therefore, the initial sample size calculation was based on the following considerations: assuming a prevalence of approximately $30 \%$ malnutrition in children $<5$ years of age, a sample size of 323 children $<5$ years of age was needed to estimate such a prevalence and a corresponding $95 \%$ CI with a precision of $\pm 5 \%$. ${ }^{63}$ To recruit this number of children, we decided to include entire households. Therefore, 1314 individuals are sufficient to estimate prevalences up to $30 \%$ within five absolute percentage points with $95 \%$ CI (in children $<5$ years of age, and their fathers and mothers). The present study consists of a subsample of the study population providing all biomarkers of interest. Even though the decreased sample size may limit the scope of the results obtained-and we acknowledge this as a limitation-we are convinced that the results, presented in the current paper, provide important information for public health stakeholders, policy makers and researchers.

The results of this research can be used for the development of interventions or policies by researchers, stakeholders and government officials. The random selection of the study participants and the standardised assessment of anthropometrical and laboratory measurements are main strengths of the present study. Moreover, we consequently applied age-specific and sex-specific cut-offs that take into account the physiological development characteristic of the young age group, rather than applying the fixed cut-offs used in the adult population. There is little information on the association of multiple obesity indices with multiple cardiometabolic risk factors in this population; hence, our study provides an important contribution towards filling this gap.

\section{CONCLUSION}

This study adds to the literature on the association of obesity with higher risks for hypertension, dyslipidemia and type 2 diabetes mellitus, but for the first time in a Zanzibari population. Based on our findings, we recommend that similar epidemiological studies including children, adolescents, adults and elderly set diabetes and/or prediabetes cut-offs of HbAlc at $\geq 6.1 \%$ and/or elevated fasting glucose at $\geq 5.6 \mathrm{mmol} / \mathrm{L}$. Where feasible, $\mathrm{BF} \%$ and WC should be used in addition to BMI for screening and monitoring for dyslipidemia and hypertension. We further conclude that there is a need for effective interventions to create awareness as well as for primary prevention strategies for cardiometabolic risks and its complications in Unguja Island, using local multidisciplinary approaches in the local language, Swahili. Additionally, there is a need for health surveillance initiatives that particularly target the age group $\geq 18$ to $<45$ years. These can also be used to help monitor prevention activities.

Acknowledgements This work was done as part of the Leibniz Graduate School SUTAS (Sustainable Use of Tropical Aquatic Systems; http://www.zmtbremen.de/SUTAS.html). This study would not have been possible without the voluntary collaboration of the Zanzibari families who participated in the extensive examinations. The authors are grateful for the support from regional and local community leaders and municipalities. The authors gratefully acknowledge the assistance from all field workers and the laboratory technicians. The publication of this article was funded by the Open Access Fund of the Leibniz Association. A poster has been presented at the 25th European Congress on Obesity (ECO), 23-26 May 2018 , Vienna,Austria: Hebestreit A, Buck C, Kelm S, Sheikh M, Brackmann K, Nyangasa MA. Association betweencardio-metabolic risk factors and body mass index, waist circumferences and body fat in a Zanzibari cross-sectional study.

Contributors AH and MAN were responsible for study design. AH, MAN and SK conducted data collection and developed study hypothesis. MAN and CB conducted statistical analyses and KB assisted in the statistical data cleaning. MAN wrote the manuscript and had primary responsibility for final content. MAN, CB, SK, MAS, KLB and $\mathrm{AH}$ critically revised the manuscript and gave final consent.

Funding The Leibniz-Gemeinschaft grant number SAW-2012-ZMT-4 supported this work.

Competing interests None declared.

Patient consent for publication Not required.

Ethics approval The study protocol was evaluated and approved by the Ethics Committees of the University of Bremen and of the Zanzibar Ministry of Health and the Zanzibar Medical Research and Ethics Committee.

Provenance and peer review Not commissioned; externally peer reviewed.

Data sharing statement The datasets generated and/or analysed during the current study are not publicly available since a follow-up study is planned.

Open access This is an open access article distributed in accordance with the Creative Commons Attribution Non Commercial (CC BY-NC 4.0) license, which permits others to distribute, remix, adapt, build upon this work non-commercially, and license their derivative works on different terms, provided the original work is properly cited, appropriate credit is given, any changes made indicated, and the use is non-commercial. See: http://creativecommons.org/licenses/by-nc/4.0/.

\section{REFERENCES}

1. World Health Organization (WHO). Fact sheet: Cardiovascular dieases (CVDs). 2016 http://www.who.int/mediacentre/factsheets/ fs317/en/ (Accessed Aug 2016).

2. Oladapo OO, Salako L, Sodiq O, et al. A prevalence of cardiometabolic risk factors among a rural Yoruba south-western Nigerian population: a population-based survey. Cardiovasc J Afr 2010;21:26-31. 
3. Boutayeb A. The double burden of communicable and noncommunicable diseases in developing countries. Trans $R$ Soc Trop Med Hyg 2006;100:191-9.

4. Mbanya JC, Motala AA, Sobngwi E, et al. Diabetes in sub-Saharan Africa. Lancet 2010;375:2254-66.

5. Sobngwi E, Mauvais-Jarvis F, Vexiau P, et al. Diabetes in Africans. Part 1: epidemiology and clinical specificities. Diabetes Metab 2001;27:628-34.

6. Christensen DL, Friis H, Mwaniki DL, et al. Prevalence of glucose intolerance and associated risk factors in rural and urban populations of different ethnic groups in Kenya. Diabetes Res Clin Pract 2009;84:303-10.

7. Obirikorang C, Osakunor DN, Anto EO, et al. Obesity and CardioMetabolic Risk Factors in an Urban and Rural Population in the Ashanti Region-Ghana: a Comparative Cross-Sectional Study. PLoS One 2015;10:e0129494.

8. Re RN. Obesity-related hypertension. Ochsner J 2009;9:133-6.

9. Carrère P, Fagour C, Sportouch D, et al. Diabetes mellitus and obesity in the French Caribbean: A special vulnerability for women? Women Health 2018;58.

10. Nayak SB, Rahming V, Raghunanan Y, et al. Prevalence of Diabetes, Obesity and Dyslipidaemia in Persons within High and Low Income Groups Living in North and South Trinidad. J Clin Diagn Res 2016;10:IC08-IC13.

11. Expert Panel on Detection, Evaluation, and Treatment of High Blood Cholesterol in Adults. Executive Summary of The Third Report of The National Cholesterol Education Program (NCEP) Expert Panel on Detection, Evaluation, And Treatment of High Blood Cholesterol In Adults (Adult Treatment Panel III). JAMA 2001;285:2486-97.

12. Daud A, Shahadan SZ. Association Between Body Mass Index and Cardiometabolic risks among malay obese adults. Clin Nurs Res 2019;28.

13. Christian $\mathrm{AH}$, Mochari $\mathrm{H}$, Mosca LJ. Waist circumference, body mass index, and their association with cardiometabolic and global risk. $J$ Cardiometab Syndr 2009;4:12-19.

14. Lee SH, Tak YJ, Yi YH, et al. Correlations between obesity indices and cardiometabolic risk factors in obese subgroups in women with severe obesity: A multicenter, cross-sectional study. Obes Res Clin Pract 2017;11:167-76.

15. Fezeu L, Balkau B, Sobngwi E, et al. Waist circumference and obesity-related abnormalities in French and Cameroonian adults: the role of urbanization and ethnicity. Int J Obes 2010;34:446-53.

16. Cole TJ, Lobstein T. Extended international (IOTF) body mass index cut-offs for thinness, overweight and obesity. Pediatr Obes 2012;7:284-94

17. Kimani-Murage EW, Kahn K, Pettifor JM, et al. The prevalence of stunting, overweight and obesity, and metabolic disease risk in rural South African children. BMC Public Health 2010;10:158.

18. Bovet $P$, Arlabosse $T$, Viswanathan B, et al. Association between obesity indices and cardiovascular risk factors in late adolescence in the Seychelles. BMC Pediatr 2012;12:176.

19. Després JP, Couillard C, Gagnon J, et al. Race, visceral adipose tissue, plasma lipids, and lipoprotein lipase activity in men and women: the Health, Risk Factors, Exercise Training, and Genetics (HERITAGE) family study. Arterioscler Thromb Vasc Biol 2000;20:1932-8.

20. Razak F, Anand S, Vuksan V, et al. Ethnic differences in the relationships between obesity and glucose-metabolic abnormalities: a cross-sectional population-based study. Int J Obes 2005;29:656-67.

21. Ruxton $\mathrm{CH}$, Reilly JJ, Kirk TR. Body composition of healthy 7-and 8-year-old children and a comparison with the 'reference child'. Int J Obes Relat Metab Disord 1999;23:1276-81.

22. Savva SC, Tornaritis M, Savva ME, et al. Waist circumference and waist-to-height ratio are better predictors of cardiovascular disease risk factors in children than body mass index. Int $J$ Obes Relat Metab Disord 2000;24:1453-8.

23. Fernández JR, Redden DT, Pietrobelli A, et al. Waist circumference percentiles in nationally representative samples of AfricanAmerican, European-American, and Mexican-American children and adolescents. J Pediatr 2004;145:439-44.

24. Jordan I, Hebestreit A, Swai B, et al. Breast cancer risk among women with long-standing lactation and reproductive parameters at low risk level: a case-control study in Northern Tanzania. Breast Cancer Res Treat 2013;142:133-41.

25. Nyangasa MA, Kelm S, Sheikh MA, et al. Design, response rates, and population characteristics of a cross-sectional study in Zanzibar, Tanzania. JMIR Res Protoc 2016;5:e235.

26. United Nations Educational Scientific and Cultural Organization (UNESCO) International Standard Classification of Education UNESCO Institute for Statistics Canada. 2011.
27. Stomfai S, Ahrens W, Bammann K, et al. Intra- and inter-observer reliability in anthropometric measurements in children. Int $\mathrm{J}$ Obes 2011;35(Suppl 1):S45-51.

28. Peplies J, Fraterman A, Scott R, et al. Quality management for the collection of biological samples in multicentre studies. Eur $J$ Epidemiol 2010;25:607-17.

29. Marfell-Jones M, Olds T, Stewart A, et al. International standards for anthropometric assessment. Potchefstroom, South Africa: International Society for the Advancement of Kinanthropometry, 2006.

30. Ahrens W, Bammann K, Siani A, et al. The IDEFICS cohort: design, characteristics and participation in the baseline survey. Int $J$ Obes 2011;35:S3-15.

31. Organization WH (WHO). BMI for age - 5 to 19 years (z-scores): World Health Organization, 2007

32. World Health Organization (WHO). The International Classification of adult underweight, overweight and obesity according to BMI. https:// www.who.int/nutrition/databases/bmi/en/

33. Nagy $P$, Kovacs E, Moreno LA, et al. Percentile reference values for anthropometric body composition indices in European children from the IDEFICS study. Int J Obes 2014;38(Suppl2):S15-S25.

34. Federation ID (IDF). The IDF consensus definition of the metabolic syndrome in children and adolescents. Brussels, Belgium: IDF Communication, 2007. ISBN 2-930229-49-7.

35. McCarthy HD, Cole TJ, Fry T, et al. Body fat reference curves for children. Int J Obes 2006;30:598-602.

36. Gallagher D, Heymsfield SB, Heo M, et al. Healthy percentage body fat ranges: an approach for developing guidelines based on body mass index. Am J Clin Nutr 2000;72:694-701.

37. Howie SR. Blood sample volumes in child health research: review of safe limits. Bull World Health Organ 2011;89:46-53.

38. Barba G, Buck C, Bammann K, et al. Blood pressure reference values for European non-overweight school children: the IDEFICS study. Int J Obes 2014;38(Suppl 2):48-56.

39. De Henauw S, Michels N, Vyncke K, et al. Blood lipids among young children in Europe: results from the European IDEFICS study. Int $J$ Obes 2014;38 Suppl 2(S2):S67-S75.

40. Peplies J, Jiménez-Pavón D, Savva SC, et al. Percentiles of fasting serum insulin, glucose, $\mathrm{HbA1c}$ and HOMA-IR in pre-pubertal normal weight European children from the IDEFICS cohort. Int $J$ Obes 2014;38(Suppl 2):S39-47.

41. Rödöö P, Ridefelt P, Aldrimer M, et al. Population-based pediatric reference intervals for $\mathrm{HbA} 1 \mathrm{c}$, bilirubin, albumin, $\mathrm{CRP}$, myoglobin and serum enzymes. Scand J Clin Lab Invest 2013;73:361-7.

42. National Institute of Health. The third report on the Diagnosis, Evaluation, and Treatment of High Blood Pressure in Children and Adolescents,. 2005.

43. National Institute of Health. The Seventh Report of the Joint National Committee on Prevention, Detection, Evaluation, and Treatment of High Blood Pressure. 2004:104.

44. Stern SE, Williams K, Ferrannini E, et al. Identification of individuals with insulin resistance using routine clinical measurements. Diabetes 2005;54:333-9.

45. Shashaj B, Luciano R, Contoli B, et al. Reference ranges of HOMAIR in normal-weight and obese young Caucasians. Acta Diabetol 2016;53:251-60.

46. von Eyben FE, Mouritsen E, Holm J, et al. Intra-abdominal obesity and metabolic risk factors: a study of young adults. Int J Obes Relat Metab Disord 2003;27:941-9.

47. Matthews DR, Hosker JP, Rudenski AS, et al. Homeostasis model assessment: insulin resistance and beta-cell function from fasting plasma glucose and insulin concentrations in man. Diabetologia 1985;28:412-9.

48. Ziraba AK, Fotso JC, Ochako R. Overweight and obesity in urban Africa: a problem of the rich or the poor? BMC Public Health 2009;9:465

49. Sodjinou R, Agueh V, Fayomi B, et al. Obesity and cardio-metabolic risk factors in urban adults of Benin: relationship with socioeconomic status, urbanisation, and lifestyle patterns. BMC Public Health 2008;8:84.

50. Keino S, Plasqui G, Ettyang G, et al. Determinants of stunting and overweight among young children and adolescents in sub-Saharan Africa. Food Nutr Bull 2014;35:167-78.

51. Ulasi II, ljoma CK, Onodugo OD. A community-based study of hypertension and cardio-metabolic syndrome in semi-urban and rural communities in Nigeria. BMC Health Serv Res 2010;10:71.

52. Sumner AE, Zhou J, Doumatey A, et al. Low HDL-Cholesterol with Normal Triglyceride Levels is the Most Common Lipid Pattern in West Africans and African Americans with Metabolic Syndrome: Implications for Cardiovascular Disease Prevention. CVD Prev Control 2010;5:75-80. 
53. Alsheikh-Ali AA, Omar MI, Raal FJ, et al. Cardiovascular risk factor burden in Africa and the Middle East: the Africa Middle East Cardiovascular Epidemiological (ACE) study. PLoS One 2014;9:e102830.

54. Garrido RA, Semeraro MB, Temesgen SM, et al. Metabolic syndrome and obesity among workers at Kanye Seventh-Day Adventist Hospital, Botswana. S Afr Med J 2009;99:331-4.

55. World Health Organization (WHO). Use of Glycated Haemoglobin (HbA1c) in the Diagnosis of Diabetes Mellitus. Use of Glycated Haemoglobin (HbA1c) in the Diagnosis of Diabetes Mellitus: Abbreviated Report of a WHO Consultation. Geneva: World Health Organization (WHO), 2011.

56. Kurtoğlu S, Hatipoğlu N, Mazıcıoğlu M, et al. Insulin resistance in obese children and adolescents: HOMA-IR cut-off levels in the prepubertal and pubertal periods. J Clin Res Pediatr Endocrinol 2010;2:100-6.

57. Caprio S, Plewe G, Diamond MP, et al. Increased insulin secretion in puberty: a compensatory response to reductions in insulin sensitivity. J Pediatr 1989;114:963-7.
58. Midha T, Idris MZ, Saran RK, et al. Prevalence and determinants of hypertension in the urban and rural population of a north Indian district. East Afr J Public Health 2009;6:268-73.

59. Gastaldelli A, Cusi K, Pettiti M, et al. Relationship between hepatic/ visceral fat and hepatic insulin resistance in nondiabetic and type 2 diabetic subjects. Gastroenterology 2007;133:496-506.

60. Ganz ML, Wintfeld N, Li Q et al. The association of body mass index with the risk of type 2 diabetes: a case-control study nested in an electronic health records system in the United States. Diabetol Metab Syndr 2014;6:50.

61. Feng RN, Zhao C, Wang C, et al. BMI is strongly associated with hypertension, and waist circumference is strongly associated with type 2 diabetes and dyslipidemia, in northern Chinese adults. $J$ Epidemiol 2012;22:317-23.

62. Noradilah MJ, Ang YN, Kamaruddin NA, et al. Assessing body fat of children by skinfold thickness, bioelectrical impedance analysis, and dual-energy X-Ray absorptiometry: a validation study among malay children aged 7 to 11 years. Asia Pac J Public Health 2016;28:74S-84.

63. Lemeshow S, Hosmer Jr D, Klar J, et al. Adequacy of sample size in health studies. 1990 